\title{
LOS DESCENDIENTES (2013): MUERTE CEREBRAL, INSTRUCCIONES PREVIAS Y EUTANASIA
}

\section{The descendants (2013): Cerebral death, living wills and euthanasia}

Emilio PINTOR HOLGUÍN, Nieves MARTíNEZ ALSINA y Benjamín HERREROS RUIZ-VALDEPEÑAS

Universidad Europea de Madrid. Facultad de Ciencias Biomédicas. Villaviciosa de Odón (España).

Autor para correspondencia: Emilio Pintor Holguín.

Correo electrónico: emilio.pintor@universidadeuropea.es

Ficha técnica

Título: Los descendientes.

Titulo original: The descendants.

País: Estados Unidos.

Año: 2011.

Director: Alexander Payne.

Música: Varios.

Fotografía: Phedon Papamichael.

Guion: Alexander Payne, Nat Faxon, Jim Rash (Novela: Kaui Hart Hemmings).

Intérpretes: George Clooney, Shailene Woodley, Amara Miller, Nick Krause, Patricia Hastie, Matthew Lillard, Judy Greer, Beau Bridges, Robert Forster, Barbara L. Southern, Mary Birdsong, Rob Huebel, Michael Ontkean, Scott Morgan, Milt Kogan.

Idioma original: inglés.

Color: color.

Duración: 110 minutos.

Género: drama, comedia dramática.
Productoras: Fox Searchlight, Ad Hominem Enterprises

Sinopsis: Matt King (George Clooney), casado y padre de dos niñas, se ve obligado a replantearse la vida cuando su mujer sufre un terrible accidente que la deja en coma. Intenta torpemente recomponer la relación con sus problemáticas hijas -la precoz Scottie, de 10 años (Amara Miller), y la rebelde Alexandra, de 17 (Shailene Woodley)- al tiempo que se enfrenta a la difícil decisión de vender las propiedades de la familia. Herederos de la realeza hawaiana y de los misioneros, los King poseen en Hawai tierras vírgenes de un valor incalculable.

\section{Enlaces:}

https://www.imdb.com/title/tt1033575/ https://www.filmaffinity.com/es/film818439. html

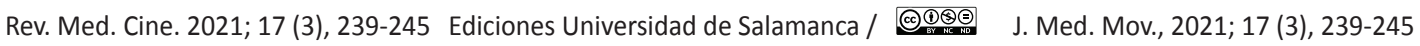




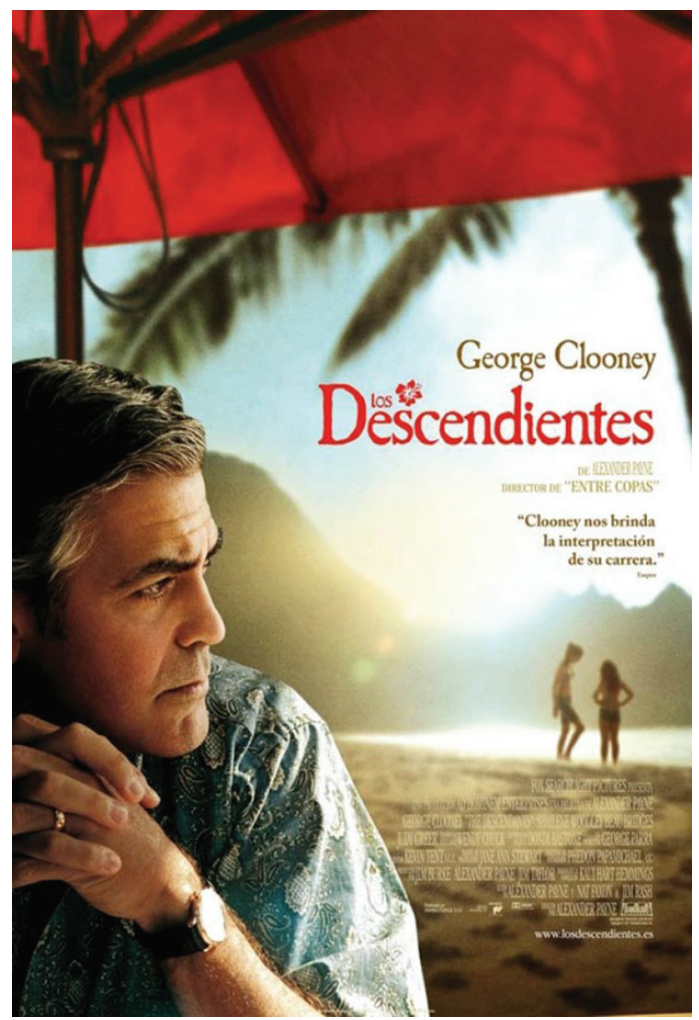

Cartel en español

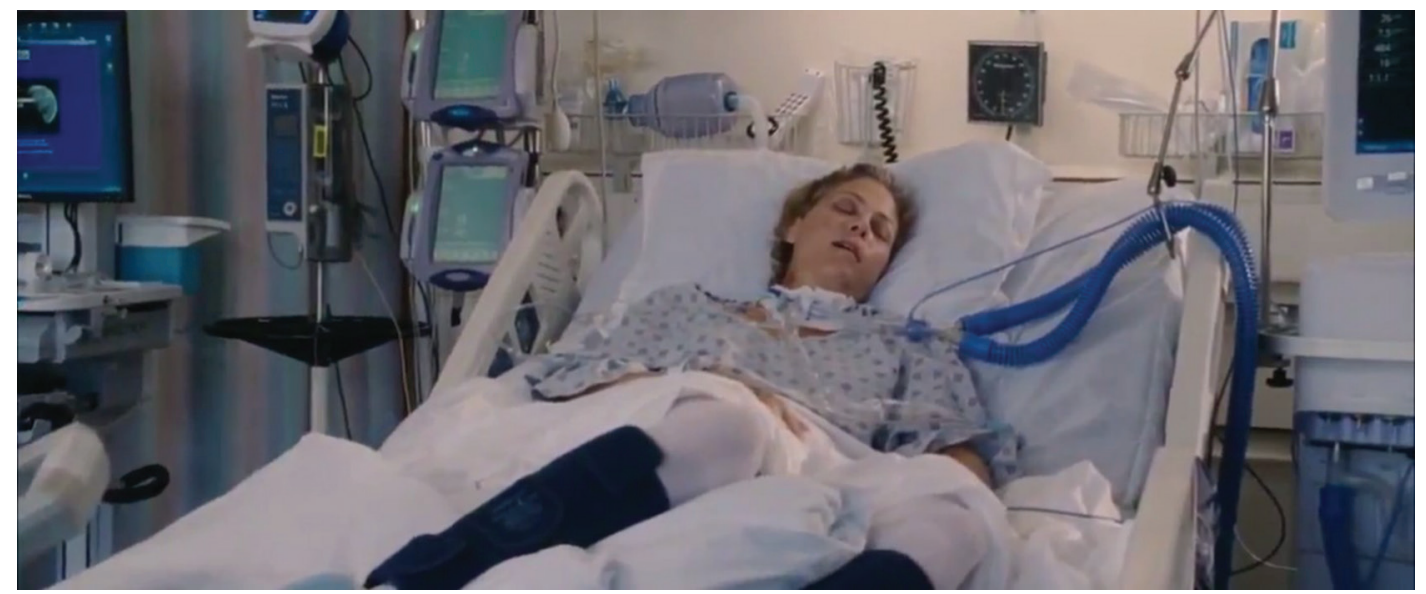

Elisabeth se encuentra en coma tras un accidente con una lancha marítima. 


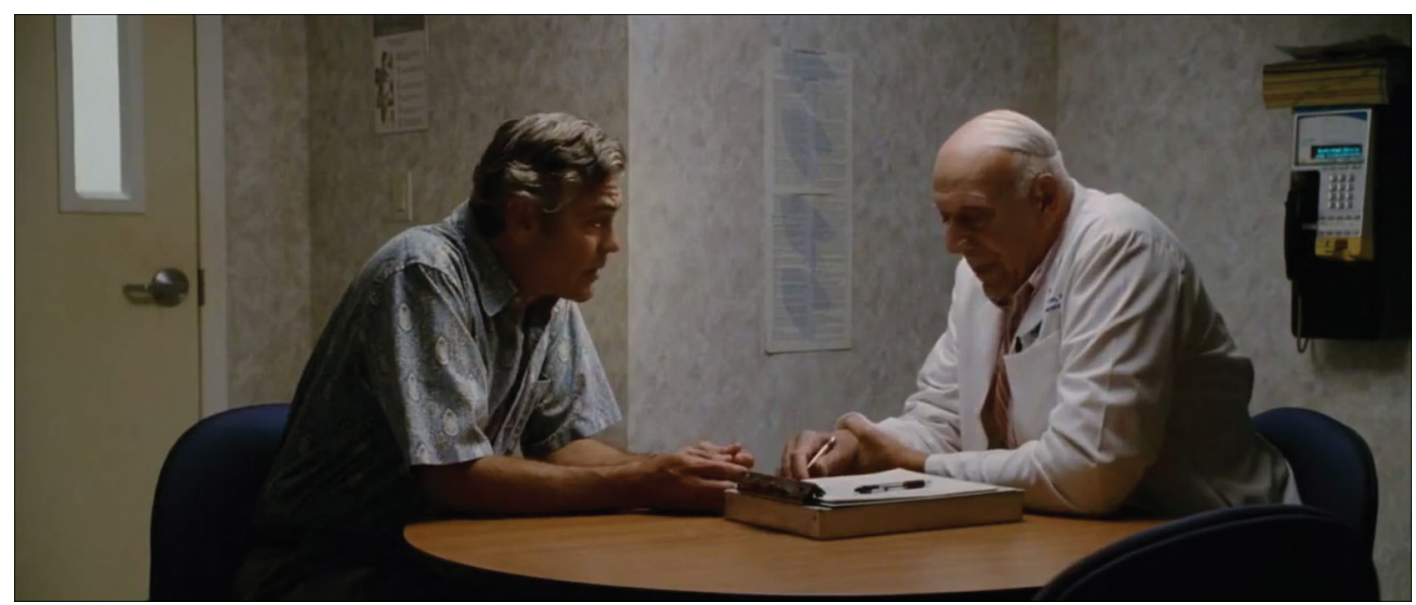

El médico informa a Matt (el marido de Elisabeth) de la irreversibilidad del coma

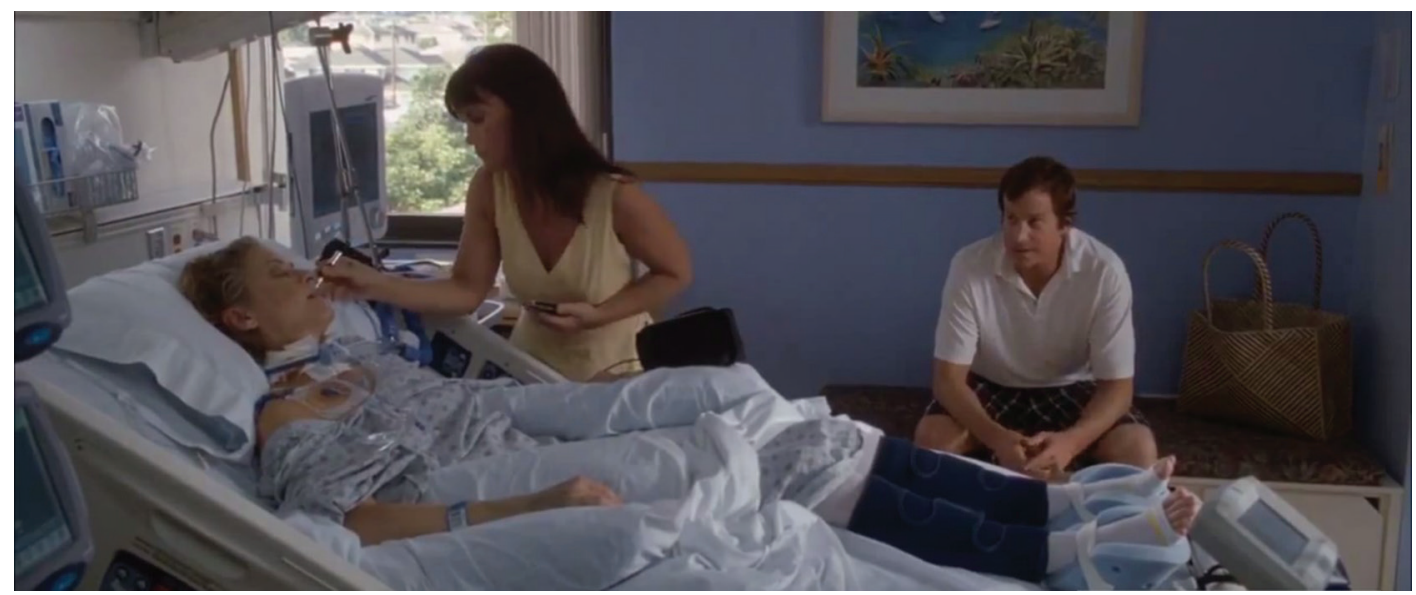

La hermana de Elisabeth la maquilla durante su estancia en el hospital

En ésta una película donde las dos tramas fundamentales son la infidelidad de Elisabeth y la decisión de si vender o no unos terrenos a una inmobiliaria en Hawai, se entremezclan con temas bioéticos muy actuales y controvertidos: eutanasia, limitación del esfuerzo terapéutico y el documento de instrucciones previas ante la situación de coma irreversible de Elisabeth.

El documento de instrucciones previas (IPS) o voluntades anticipadas surgió en Estados Unidos, dentro de los denominados "living wills", como método para limitar la actuación médica en aquellos casos en los que el paciente no pudiera expresar qué cuidados querría recibir ante una situación de incapacidad ${ }^{1}$. En España en el año 2002 se promulgó la Ley 41/2002 reguladora de la autonomía del paciente ${ }^{2}$, donde se exponían los derechos y deberes respecto la relación clínica tanto de pacientes como de profesionales sanitaros. En dicha Ley se especificaba cómo los 


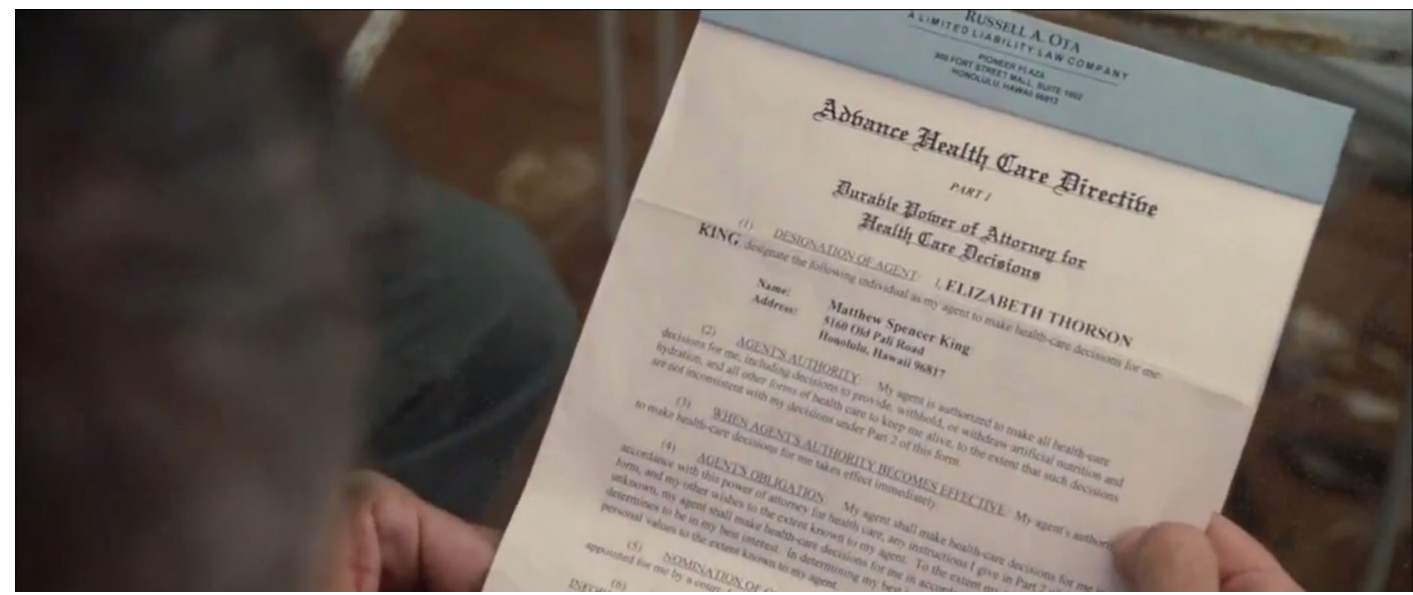

Matt enseña a su suegro el documento de Instrucciones Previas (IPs) firmado por Elisabeth

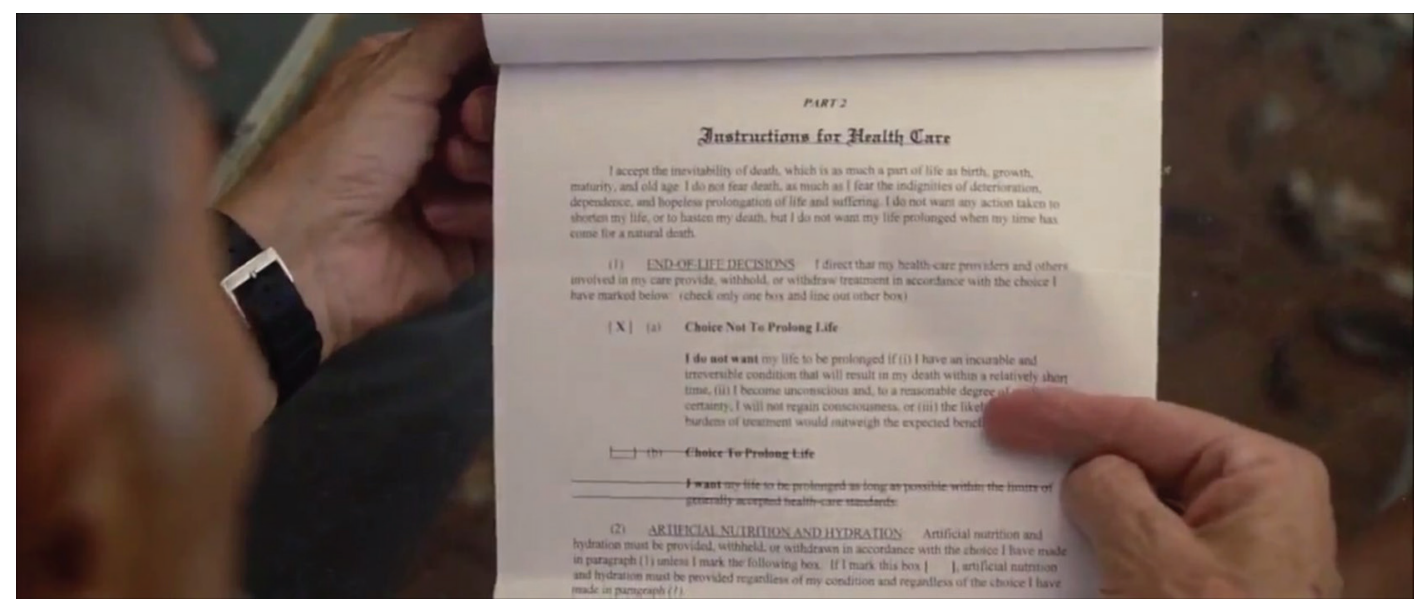

Instrucciones previas de Elisabeth: Decisiones al final de la vida: "En caso de enfermedad incurable e irreversible no quiero que mi vida sea prolongada."

pacientes podían ejercer su autonomía, incluido el derecho a expresar su voluntad de forma diferida a través de las IPs. La Ley 41/2002 formaba parte de una estrategia de armonización en legislación sanitaria con el resto de los Estados miembros de la Unión Europea (UE) y de acuerdo con el Convenio de Oviedo (Oviedo, 4 de abril de
1997) del Consejo de Europa, ratificado por los 19 Estados miembros de la UE. Dicho Convenio fue ratificado por España el uno de enero de 2000, de lo que resultó en una legislación sobre las IPs en varias Comunidades Autónomas (Andalucía 1998, Cataluña 2000, Extremadura 2001) antes de la mencionada Ley estatal 41/2002. 


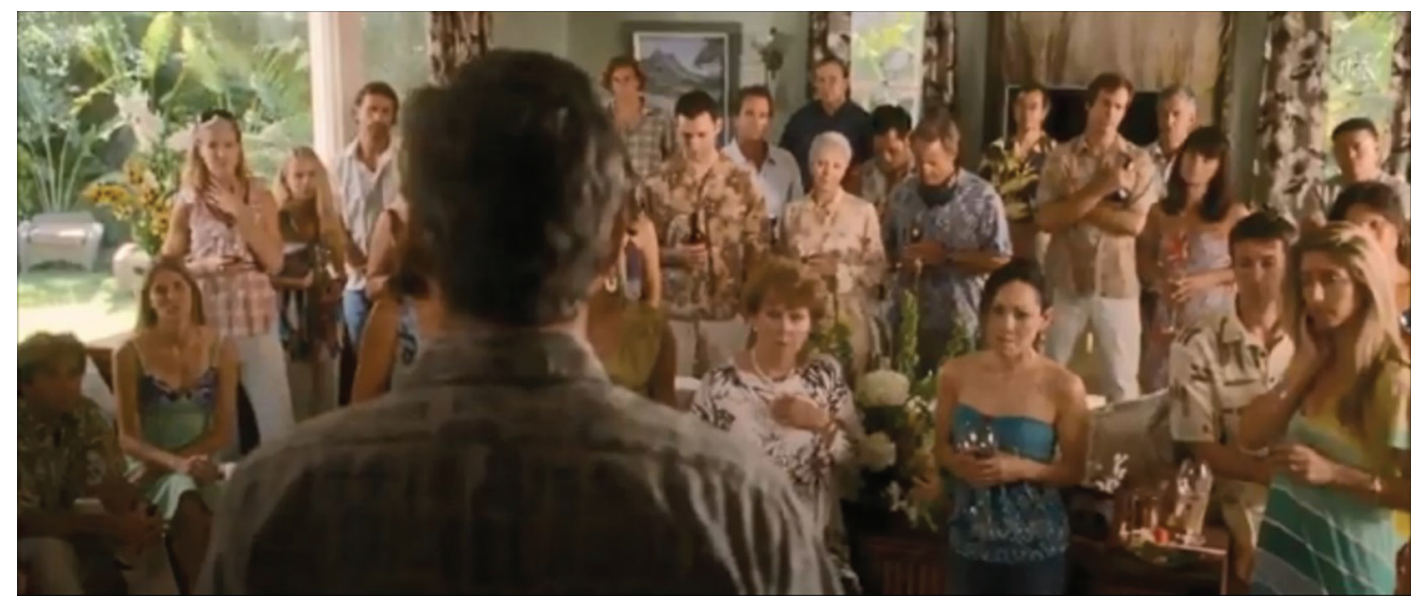

Matt reúne a los familiares y amigos de Elisabeth para explicarles la situación y para que vayan a "despedirse de ella".

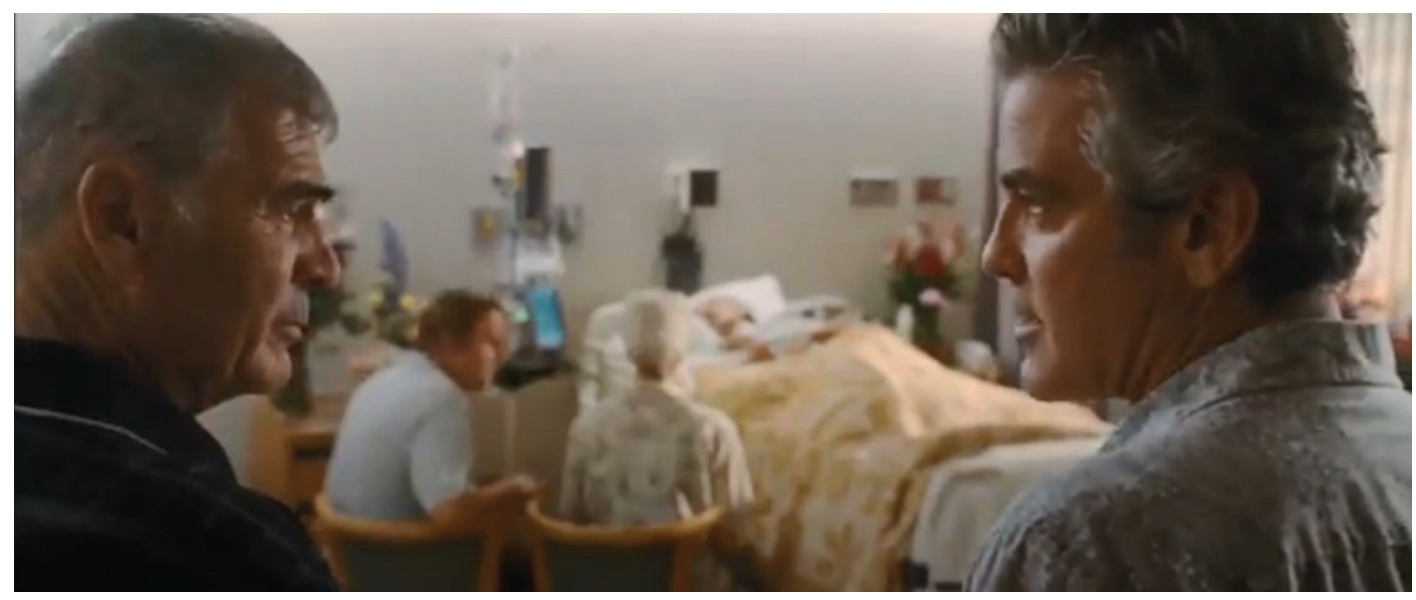

Los padres y el hermano de Elisabeth acuden al hospital a verla por última vez.

El sistema sanitario español está descentralizado, por lo que la gestión de las IPs depende de las Comunidades Autónomas. En la Ley 41/2002 se especifica que las Comunidades Autónomas deben realizar una regulación sobre las IPs en su territorio $y$, como consecuencia, todas las Comunidades han elaborado su propia norma sobre las IPs. Sin embargo, para que puedan ser conocidas y respetadas en todo el territorio nacional (por ejemplo, para que un paciente que las ha registrado en Madrid las vea respetadas si reside en Valencia), en 2007 se desarrolló el Real Decreto 124/2007, que regula el Registro Nacional de IPs ${ }^{3}$. 


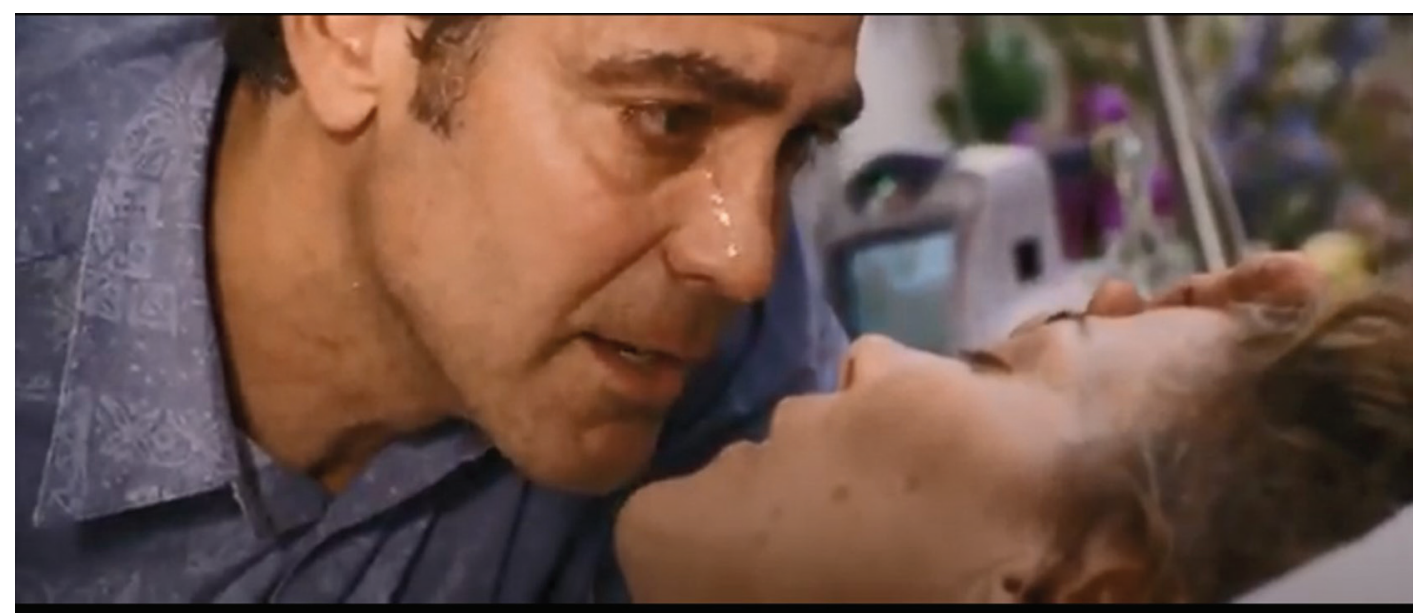

Llega el momento de cumplir las IPs: apagar el soporte vital de Elisabeth. Matt se despide de ella.

A pesar de existir una legislación sobre IPs a nivel nacional y en todas las Comunidades Autónomas, el uso de las IPs en la práctica clínica es escaso y el número de ciudadanos que expresan sus deseos sobre su futuro plan de cuidados a través de unas IPs es muy bajo. Según datos del Ministerio de Sanidad (Registro Nacional de Datos de IPs) ${ }^{4}$, desde la aprobación de las Leyes promulgadas en España (2002 a 2007), las IPs registradas han pasado de 36.289 en 2007 a 314.011 en 2020. Este aumento en números absolutos representa solo al $0,66 \%$ de la población. Las Comunidades que tienen registrados más documentos son Cataluña, Andalucía y Madrid (las mas pobladas) y las que tienen mayores tasas por 1.000 habitantes son Navarra, País Vasco y Cataluña. La implementación de una legislación nacional sobre IPs en España ha aumentado el número de estudios empíricos sobre las IPs en la última década ${ }^{5,6}$, explorando el papel de los pacientes, de los allegados y representantes y de los profesionales sanitarios.

Los estudios en España sobre las IPs, realizados en profesionales sanitarios, pacientes y sus allegados, muestran cómo a pesar del gran desarrollo legislativo que se ha hecho, todavía hay un gran desconocimiento, su puesta en práctica es excesivamente farragosa y su relevancia en la toma de decisiones es muy escaso, lo que puede generar situaciones en las que no se respeten los deseos expresados anticipadamente por los enfermos. No obstante, en profesionales y ciudadanos cada vez hay mayor interés hacia las IPs y en general hay una actitud positiva tanto hacia las IPs como por planificación anticipada de las decisiones.

Se considera que las IPs son una ayuda para la toma de decisiones, mejoran la relación clínica y fortalecen la confianza. Entre los usuarios, se han detectado actitudes más en enfermos con procesos crónicos y enfermedades terminales, en los familiares de enfermos ingresados en unidades de cuidados intensivos y en los cuidadores de enfermos crónicos y terminales, lo que da idea de cómo la cercanía con el sufrimiento sensibiliza en la necesidad planificar la forma de morir.

Pero esto no significa que haya que llegar a estas situaciones para planificar el final de la vida. El trabajo desde atención primaria, tanto para difundir las IPs como en su implementación, 
resulta primordial. La falta de formación y el escaso número de IPs entre los pacientes españoles lleva a que la mayoría de los profesionales no se plantee la posibilidad de que un paciente pueda tenerlas redactadas. Los usuarios consideran, además, que no siempre se respetan, lo que concuerda con la creencia de muchos pacientes de que tener redactadas unas IPs no cambiaría la actitud del médico y con la opinión de los representantes, que señalan que no siempre se les consulta al tomar decisiones. Esto puede ser reflejo de la tradición paternalista en medicina, de la que aún quedan resquicios en la medicina española.

A pesar de las posibles mejoras que puedan implantarse, las IPs tienen problemas imposibles del solventar: son documentos estáticos y en ocasiones difíciles de interpretar, su consulta puede resultar compleja, etcétera. Para solventar dichas carencias una alternativa es la Planificación Anticipada de las Decisiones sanitarias. La PAD es un medio más eficaz y completo que las IPs para respetar la voluntad del paciente, sin olvidar que en el proceso de planificación se puede incluir la realización de unas IPs como una herramienta más para que el enfermo pueda dejar constancia de sus deseos.

\section{Referencias}

1. Martínez Urionabarrenetxea K. Reflexiones sobre el testamento vital (I y II). Aten Primaria. 2003;31(1): 52-4.

2. Basic Law $41 / 2002$ of 14 November, governing the autonomy of the patient and rights and obligations with regard to clinical information and documentation.

3. Real Decreto $124 / 2007$, de 2 de febrero, por el que se regula el Registro nacional de instrucciones previas y el correspondiente fichero automatizado de datos de carácter personal. BOE núm 40; pág. 6591-3, de 15 de febrero de 2007.

4. Ministerio de Sanidad y Consumo «BOE» núm. 40, de 15 de febrero de 2007.

5. Simon Lorda P, Tamayo Velazquez MI, Barrio Cantalejo IM. Advance directive in Spain. Perspecitves from a medical bioethicist's approach. Bioethics. 2008;22(6):346-54.

6. Flordelís Marco F. Testamento vital en las historias clínicas. Trabajo Social y Salud. 2000;37:85-104. 